Check for updates

Cite this: RSC Adv., 2017, 7, 48853

\title{
Molybdenum trioxide thin film recombination barrier layers for dye sensitized solar cells
}

\author{
Aditya Ashok, S. N. Vijayaraghavan, (D) Shantikumar V. Nair* \\ and Mariyappan Shanmugam (DD*
}

A physical vapor deposition based molybdenum trioxide $\left(\mathrm{MoO}_{3}\right)$ thin film is demonstrated as an efficient reverse-electron recombination barrier layer (RBL) at the fluorine doped tin oxide (FTO)/titanium dioxide $\left(\mathrm{TiO}_{2}\right)$ interface in dye sensitized solar cells (DSSCs). Thin films of $\mathrm{MoO}_{3}$ show an average optical transmittance of $\sim 77 \%$ in a spectral range of 350-800 nm with bandgap value of $\sim 3.1 \mathrm{eV}$. For an optimum thickness of $\mathrm{MoO}_{3}$, deposited for 5 minutes, the resulting DSSCs showed $15 \%$ enhancement in efficiency $(\eta)$ compared to the reference DSSC which did not use $\mathrm{MoO}_{3} \mathrm{RBL}$; this suggests that $\mathrm{MoO}_{3}$ is effectively suppressing interfacial recombination at the $\mathrm{FTO} / \mathrm{TiO}_{2}$ interface. Further, increasing the thickness of $\mathrm{MoO}_{3} \mathrm{RBL}$ at the $\mathrm{FTO} / \mathrm{TiO}_{2}$ interface (20 minutes deposition) is observed to impede charge transport, as noticed with $55 \%$ reduction in $\eta$ compared to the reference DSSC. Thin film $\mathrm{MoO}_{3} \mathrm{RBL}$ with an optimum thickness value at the $\mathrm{FTO} / \mathrm{TiO}_{2}$ interface efficiently blocks the leaky transport pathways in the mesoporous $\mathrm{TiO}_{2}$ nanoparticle layer and facilitates efficient charge transport as confirmed by electrochemical impedance spectroscopy.

Received 14th August 2017

Accepted 12th October 2017

DOI: $10.1039 / \mathrm{c} 7 \mathrm{ra0} 8988 \mathrm{k}$

rsc.li/rsc-advances varying the particle size. In general, unsaturated atomic bonds (dangling bands) on the surface of $\mathrm{TiO}_{2}$ result as surface states which are considered to be defect states which actively play a detrimental role on charge transport. ${ }^{24,25}$ While surface area of $\mathrm{TiO}_{2}$ is increased, the density of surface states that are present on the surface also increases. This situation offers certain advantages to DSSCs optically and disadvantages electrically. The hole conductor, often liquid electrolyte, diffuses into the bulk of dye coated $\mathrm{TiO}_{2}$ to form the junction through which the dye molecules are regenerated. Thus, the interfaces between $\mathrm{TiO}_{2} /$ dye/electrolyte are important at which significant photovoltaic processes occur. While the $\mathrm{TiO}_{2}$ allows the electrolyte to form the junction throughout the bulk, it might make an electrical contact with transparent electrode which is essentially an unwanted short circuit and the resulting solar cell will not be functional. To avoid such unwanted situation, a thinner $\mathrm{TiO}_{2}$ layer of smaller particle size was introduced into the transparent electrode and $\mathrm{TiO}_{2}$ interface, called compact layer. ${ }^{26}$ The ions in the liquid electrolyte can diffuse into the mesoporous $\mathrm{TiO}_{2}$ but will be blocked by compact $\mathrm{TiO}_{2}$ due to the smaller size nanoparticles. Other than $\mathrm{TiO}_{2}$ nanoparticles, various other materials, such as $\mathrm{ZnO}$ and $\mathrm{HfO}_{2}$, have been explored for compact layer applications and observed to enhance the performances significantly. ${ }^{27,28}$ All these studies show that compact layers in DSSCs should be highly dense, pin hole-free, and less defective to block the reverse electron transport from the transparent electrode to the electrolyte in which holes will be available for recombination. Further, effect of metal doping in compact layers, for example $\mathrm{Li}$ and $\mathrm{Nb}$, and surface treatment 
by $\mathrm{TiCl}_{4}$ were examined to control the recombination loss in DSSCs. ${ }^{29-31}$ All these studies show a major requirement to control the photo-generated electron recombination at the interface of transparent electrode and mesoporous $\mathrm{TiO}_{2}$. Suppressing such interfacial recombination in DSSCs, in general, is one of the ways to improve the performance of resulting DSSCs.

Molybdenum trioxide $\left(\mathrm{MoO}_{3}\right)$, an orthorhombic crystal structured material, exhibits an optical bandgap of $\sim 3.1 \mathrm{eV}$ with a work function $5.3 \mathrm{eV}$. Efficient hole injection characteristics of $\mathrm{MoO}_{3}$ have been studied in transparent conductor oxide (TCO) and organic hole transporting materials. ${ }^{32-34}$ Physical/chemical vapor deposited $\mathrm{MoO}_{3}$ thin films have shown promising applications in gas sensing and electro-chromic devices. ${ }^{35,36} \mathrm{~F}$. Cheng et al. has recently reported the organic solar cells employing $\mathrm{MoO}_{3}$ based hole transport layer. ${ }^{37}$ Various material processing methods including physical/chemical vapor deposition, flash evaporation, ion beam and wet-chemical based techniques are commonly used to deposit $\mathrm{MoO}_{3} \cdot{ }^{38-40}$

In this work, capability of thin film $\mathrm{MoO}_{3}$, deposited by reactive DC sputtering, as a reverse electron RBL is examined in DSSCs. Further, effect of deposition time (thickness) on the performance of DSSCs was studied and it was observed that there is an optimum $\mathrm{MoO}_{3}$ thickness to suppress the interfacial recombination by facilitating the electron transport and blocking the holes at $\mathrm{FTO} / \mathrm{TiO}_{2}$ interface. The presented results also clarify the increased thickness of $\mathrm{MoO}_{3}$ impede the charge transport by establishing an energy barrier at $\mathrm{FTO} / \mathrm{TiO}_{2}$ interface. Thus, $\mathrm{MoO}_{3}$ thin film can be considered as a dense and pin-hole free RBL for DSSCs.

\section{Experimental section}

\section{(i) Fabrication of reference DSSCs}

To explore and prove the concept of $\mathrm{MoO}_{3}$ thin film based RBLs, two kinds of DSSCs were fabricated (1) DSSCs employing mesoporous $\mathrm{TiO}_{2}$ as an electron transport layer, coated directly on FTO electrode. This DSSC was considered as a reference cell to evaluate the effect of $\mathrm{MoO}_{3}$ RBLs. (2) DSSCs with $\mathrm{MoO}_{3}$ thin films as RBLs coated on FTO. To fabricate the reference DSSC, $\sim 10 \mu \mathrm{m}$ thick colloidal $\mathrm{TiO}_{2}$ nanoparticle layer was coated onto FTO coated glass substrate by doctor blade method followed by annealing at $450{ }^{\circ} \mathrm{C}$ for 1 hour. The $\mathrm{TiO}_{2}$ coated on FTO was sensitized by Ruthenium based N719 dye for 12 hours. The dye solution used in this study was prepared by dissolving $0.5 \mathrm{mM}$ of N719 in 1:1 mixture of acetonitrile and tertbutyl alcohol solution. Commercially available Iodolyte AN-50 was used as a hole transporting layer. A $50 \mathrm{~nm}$ Pt thin film was used as a counter electrode. Dye coated $\mathrm{TiO}_{2}$ photo-anode and the $\mathrm{Pt}$ coated counter electrode was coupled using a parafilm spacer and the electrolyte was injected between the electrodes through a channel made on a parafilm spacer and sealed before measurements. No $\mathrm{TiCl}_{4}$ treatment and $\mathrm{TiO}_{2}$ blocking layer were used in this work to examine the effect only due to $\mathrm{MoO}_{3}$ in DSSC performance without considering any other surface modifications that are usually followed in DSSC fabrication procedure.

\section{(ii) Fabrication of $\mathrm{MoO}_{3}$ coated DSSCs}

To demonstrate $\mathrm{MoO}_{3}$ as a potential candidate for RBL application in DSSCs, thin films of $\mathrm{MoO}_{3}$ were deposited onto FTO by reactive DC sputtering using oxygen as a bombarding gas and molybdenum as a metal target. Oxygen flow rate was kept at 30 sccm and all $\mathrm{MoO}_{3}$ films were deposited at $200{ }^{\circ} \mathrm{C}$. The films were deposited for 5, 10, 15 and 20 minutes to study the effect of thickness of $\mathrm{MoO}_{3}$ on the performance of DSSCs. The $\mathrm{MoO}_{3}$ deposited FTO electrodes were used to coat mesoporous $\mathrm{TiO}_{2}$ electron transport layer. The remaining fabrication procedure is the same as explained in case of reference DSSC fabrication.

\section{(iii) Fabrication of DSSCs with $\mathrm{TiCl}_{4}$ coated FTO}

Titanium tetrachloride $\left(\mathrm{TiCl}_{4}\right)$ stock solution was freshly prepared as reported by S. Ito et al. ${ }^{\mathbf{4 1}}$ FTO coated glass substrates were dipped in $\mathrm{TiCl}_{4}$ for 30 minutes at $70{ }^{\circ} \mathrm{C}$ and annealed at $450{ }^{\circ} \mathrm{C}$ for 30 minutes. In order to fabricate DSSCs using $\mathrm{TiCl}_{4}$ coated FTO, the mesoporous $\mathrm{TiO}_{2}$ was coated onto $\mathrm{FTO} / \mathrm{TiCl}_{4}$ electrode and annealed at $450{ }^{\circ} \mathrm{C}$ for 1 hour. The remaining fabrication steps are the same as explained in reference DSSC fabrication.

\section{(iv) Materials and DSSC characterizations}

Morphology of the $\mathrm{TiO}_{2}$ and $\mathrm{MoO}_{2}$ thin film samples were analyzed in a scanning electron microscope (SEM) using JSM6490-LA from JEOL. X-ray photoelectron spectroscopy (XPS) was used to confirm the thin films of $\mathrm{MoO}_{3}$ using Kratos Analytical unit. Optical characteristics of the $\mathrm{MoO}_{3}$ films, FTO and N719 were studied by Perkin Elmer Lambda-750 UV-Visible spectrometer. The current density-voltage $(J-V)$ measurements of the DSSCs were performed under AM1.5 illumination level using Newport Oriel Class A solar simulator and a digital source meter (Keithley 2400). Electrochemical Impedance Spectroscopic (EIS) studies were performed in the reference and the DSSCs employing $\mathrm{MoO}_{3}$ RBLs under dark condition using Autolab electrochemical work station.

\section{Results and discussion}

Fig. 1 shows SEM images illustrating the surface morphologies of (a) $\mathrm{TiO}_{2}$ nanoparticles coated onto FTO electrode and (b-e) reactive DC sputtered $\mathrm{MoO}_{3}$ RBLs on the FTO substrates. The $\mathrm{TiO}_{2}$ nanoparticle layer is observed to be highly porous and having $\sim 20 \mathrm{~nm}$ nanoparticles distributed randomly. The $\mathrm{TiO}_{2}$ nanoparticle network is well connected through which photogenerated electrons diffuse to reach the FTO electrode. Physical connectivity among the nanoparticles, particle distribution and electrical conductivity play a significant role on charge transport in the bulk $\mathrm{TiO}_{2}$ nanoparticle network. However, it is obvious that the electrolyte ions can diffuse through such porous layer and this situation is expected to result in unwanted electrical short with FTO electrode. While the porosity of $\mathrm{TiO}_{2}$ nanoparticle layer assists in dye loading, electrolyte diffusion towards FTO electrode is an unwanted situation in DSSCs. Fig. 1(b-e) show surface morphologies of $\mathrm{MoO}_{3}$ at different magnifications. These morphologies assert there is no porosity 

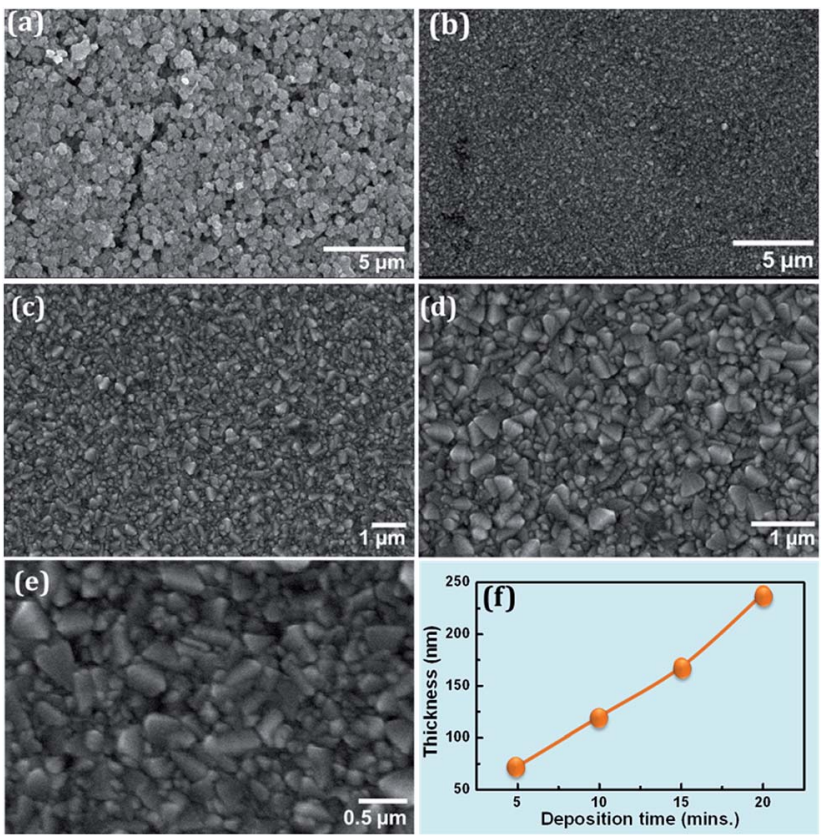

Fig. 1 SEM images showing (a) porous $\mathrm{TiO}_{2}$ exhibiting randomly distributed $\mathrm{TiO}_{2}$ nanoparticles in a porous network, (b-e) show $\mathrm{MoO}_{3}$ RBL coated onto FTO at different magnifications show a better compactness through which diffusion of electrolyte was significantly suppressed and (f) deposition time vs. thickness of $\mathrm{MoO}_{3}$ obtained.

in the bulk as can be observed in the $\mathrm{TiO}_{2}$ nanoparticle film shown in Fig. 1(a). This is a major requirement for a material to performance as an efficient RBL in DSSCs to suppress the electrolyte diffusion towards the FTO. A pinhole-free thin film with no porosity is expected to block the electrolyte ions and avoid direct electrical contact between the hole transport layer and electron-collecting electrode. The $\mathrm{MoO}_{3}$ particles distributed in the samples were random in sizes but their packing fraction is observed to be higher than that of $\mathrm{TiO}_{2}$ nanoparticle layer, which is an essential factor to effectively block the hole transporting electrolyte from touching the FTO. Fig. 1(b, c, $\mathrm{d}$ and e) obtained under the magnifications of $5000 \times, 10000 \times$, $20000 \times$ and $30000 \times$ respectively.

While the lower magnification images show uniform distribution of particles, the image obtained at $30000 \times$ illustrates the surface is covered by tightly bound $\mathrm{MoO}_{3}$ particles of different size distribution. Larger size particles are observed to be on the surface while the gap between the larger particles are perfectly filled with relatively smaller size $\mathrm{MoO}_{3}$ particle distribution as can be observed in Fig. 1(e). Thus, the $\mathrm{MoO}_{3}$ film is highly dense due to the filling effect of smaller size particles among the distribution larger size particles. In general, colloidal $\mathrm{TiO}_{2}$ nanoparticles are used for compact layer applications in DSSCs. However, the colloidal layer also is expected to have porosity to certain extent due to its nature of morphology. Physical vapor deposition, in general, offers highly uniform, dense and compact thin films through which electrolyte diffusion is not expected due to the tightly arranged particle distribution as can be seen in Fig. 1(b-e). It is very obvious that presence of such highly compact metal oxide layer at the interface of $\mathrm{FTO} / \mathrm{TiO}_{2}$ act as a barrier for any kind of liquid materials, in this case it is hole transporting electrolyte. This particular sample illustrated in Fig. 1(b-e) was deposited at $200{ }^{\circ} \mathrm{C}$ for 20 minutes. Profilometer measured thickness values of the samples deposited for $5,10,15$ and 20 minutes were $70 \mathrm{~nm}, 120 \mathrm{~nm}, 170 \mathrm{~nm}$ and $240 \mathrm{~nm}$ respectively as shown in Fig. 1(f).

XPS studies were performed on $\mathrm{MoO}_{3}$ thin film deposited on glass substrate to confirm and examine various constituting elements, particularly molybdenum and oxygen. Fig. 2(a) shows XPS survey spectrum obtained on the $\mathrm{MoO}_{3}$ sample illustrating characteristic peaks of molybdenum, oxygen and carbon in the binding energy range of $0-700 \mathrm{eV}$. The wide scan survey spectrum showed Mo 4p at $42 \mathrm{eV}, \mathrm{Mo} 3 \mathrm{~d}$ at $235 \mathrm{eV}, \mathrm{C} 1 \mathrm{~s}$ at $284 \mathrm{eV}$ and $\mathrm{O} 1 \mathrm{~s}$ at $529 \mathrm{eV}$. Doublet core levels of $\mathrm{MoO}_{3}$ shown in the survey spectrum was further studied through a high resolution scan. Fig. 2(b and c) show high resolution XPS characteristic spectra of Mo $3 \mathrm{~d}$ and $\mathrm{O} 1 \mathrm{~s}$ peaks respectively. The spin orbit doublet of $\mathrm{MoO}_{3}$ is further deconvoluted as shown in Fig. 2(c), confirming the Mo $3 \mathrm{~d}_{5 / 2}$ and Mo $3 \mathrm{~d}_{3 / 2}$ observed at $232.6 \mathrm{eV}$ and $235.8 \mathrm{eV}$ correspond to $\mathrm{Mo}^{6+}$ in stoichiometric $\mathrm{MoO}_{3}$ film. The $\mathrm{O} 1 \mathrm{~s}$ peak is comprised of two different contributions as shown in deconvoluted high resolution scan in Fig. 2(c). The peak fit identified $528.5 \mathrm{eV}$ corresponding to $\mathrm{Mo}=\mathrm{O}$ bonding between
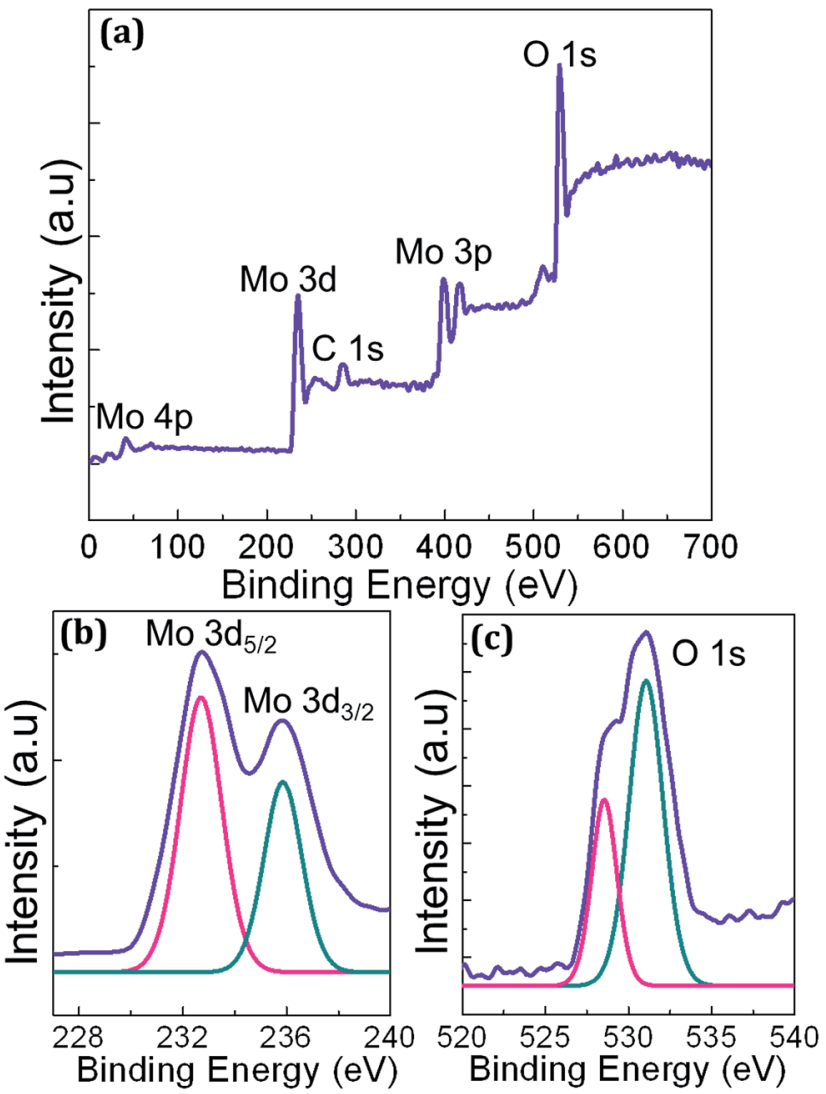

Fig. 2 XPS spectra of PVD grown $\mathrm{MoO}_{3}$ thin film sample showing (a) wide scan survey spectrum and high resolution scans of (b) Mo $3 \mathrm{~d}$ and (c) $O 1 \mathrm{~s}$ characteristic peaks. 
molybdenum and oxygen while $531.0 \mathrm{eV}$ represents the $\mathrm{OH}$ group adsorbed on the surface of the $\mathrm{MoO}_{3}$ film.

Fig. 3 shows UV-Visible optical spectroscopic characteristics of $\mathrm{MoO}_{3}$ samples along with the FTO used for DSSC fabrication illustrating absorbance and transmittance in a wavelength window of $350-800 \mathrm{~nm}$. The $\mathrm{MoO}_{3}$ samples exhibit slightly more absorbance and less transmittance compared to the FTO and their optical absorbance and transmittance characteristics overlap on each other as shown in Fig. 2(a) due to the smaller variation in their thickness offered by the duration of deposition which are 5, 10, 15 and 20 minutes. Fig. 3(b and c) shows the magnified view of the absorbance and transmittance spectra shown in Fig. 3(a) in a spectral region of 350-700 nm showing the smaller variation clearly. The FTO, a wide bandgap metal oxide transparent conductor, exhibits no photonic absorption in the spectral window of $350-800 \mathrm{~nm}$. This confirms that incoming photons don't lose their energy at the electrode while reaching the photo-active material; in this case it is N719. Further, UV-Visible optical characteristics of $\mathrm{MoO}_{3}$ films also exhibit similar features confirming that photonic loss at the RBL also will be very minimum. Thus, optical loss at FTO electrode and $\mathrm{MoO}_{3} \mathrm{RBL}$ is considered to be insignificant. Further, absorption coefficient $(\alpha)$ values for all $\mathrm{MoO}_{3}$ samples were calculated from the transmittance spectra and used to
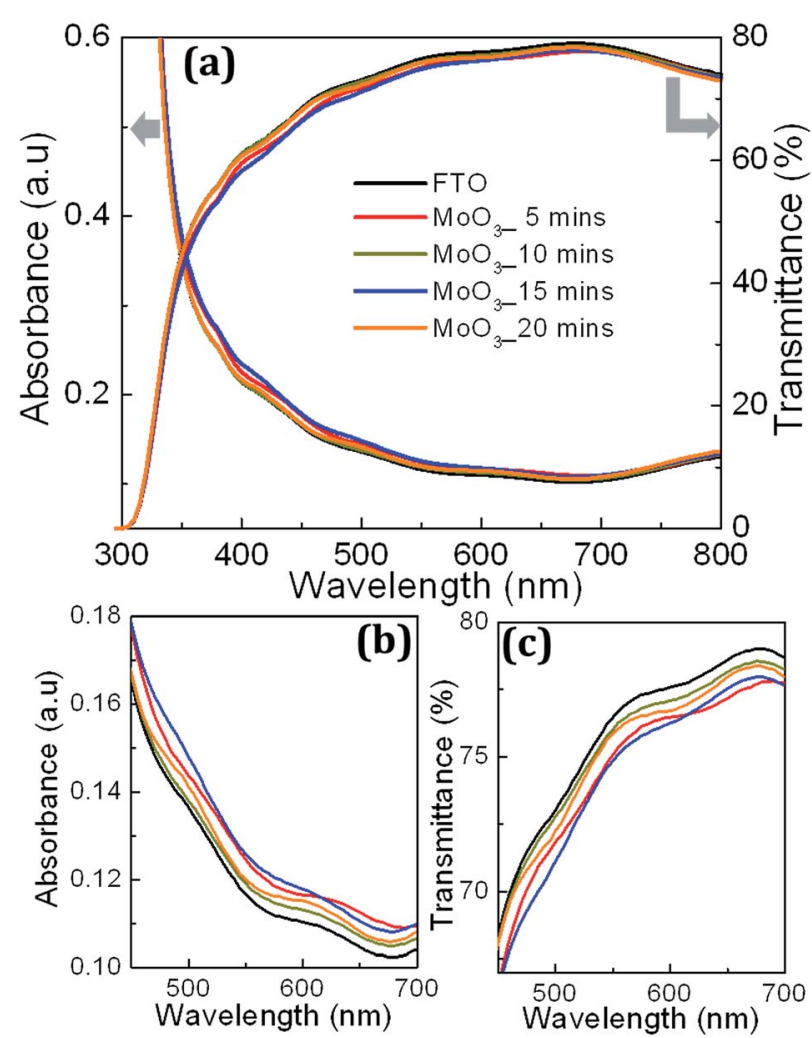

Fig. 3 (a) Optical absorption and transmittance spectra of FTO along with $\mathrm{MoO}_{3}$ samples deposited on FTO for 5, 10, 15 and 20 minutes show overlap in the characteristics due to the slight variation (the arrows indicate the axes for the absorbance and transmittance characteristics). (b and c) Magnified absorbance and transmittance showing clear variation in a spectral window of $450-700 \mathrm{~nm}$. calculate optical bandgap values through Tauc plot technique shown in Fig. 4(a). The characteristic spectra $(\alpha h \nu)^{1 / 2} v s$. energy of incoming photons at $(\alpha h \nu)^{1 / 2}=0$ yields optical bandgap values of $\mathrm{MoO}_{3}$ samples deposited for 5, 10, 15 and 20 minutes.

The extracted bandgap values for all samples were about $\sim 3.1 \mathrm{eV}$ with $\sim 0.1 \mathrm{eV}$ variation for the sample processed for 20 minutes. The observed insignificant variation in the bandgap value $(0.1 \mathrm{eV})$, can be ignored due to the cause of longer duration of oxygen flow which alters the band structure slightly. The value of optical bandgap obtained for $\mathrm{MoO}_{3}$ samples confirm that incoming solar photons in a wavelength range of 350$800 \mathrm{~nm}$ do not interact with $\mathrm{MoO}_{3}$. It implies that $\mathrm{MoO}_{3}$, as a RBL in DSSCs, does not block the visible spectral photons which supposed to be absorbed by N719 used as a major photoactive dye layer on mesoporous $\mathrm{TiO}_{2}$. To illustrate the "optical compatibility" between the $\mathrm{MoO}_{3}$ and N719, absorbance characteristics of $\mathrm{MoO}_{3}$ and N719 dye are compared as shown in Fig. 4(b). The two major peaks observed at $385 \mathrm{~nm}$ and $525 \mathrm{~nm}$ in the absorbance spectrum confirm N719 while the near-flat characteristic represents $\mathrm{MoO}_{3}$ due to the optical bandgap of $3.1 \mathrm{eV}$ shown in Fig. 4(a). It is obvious that $\mathrm{MoO}_{3}$ is not expected to absorb solar photons in the visible spectral window as
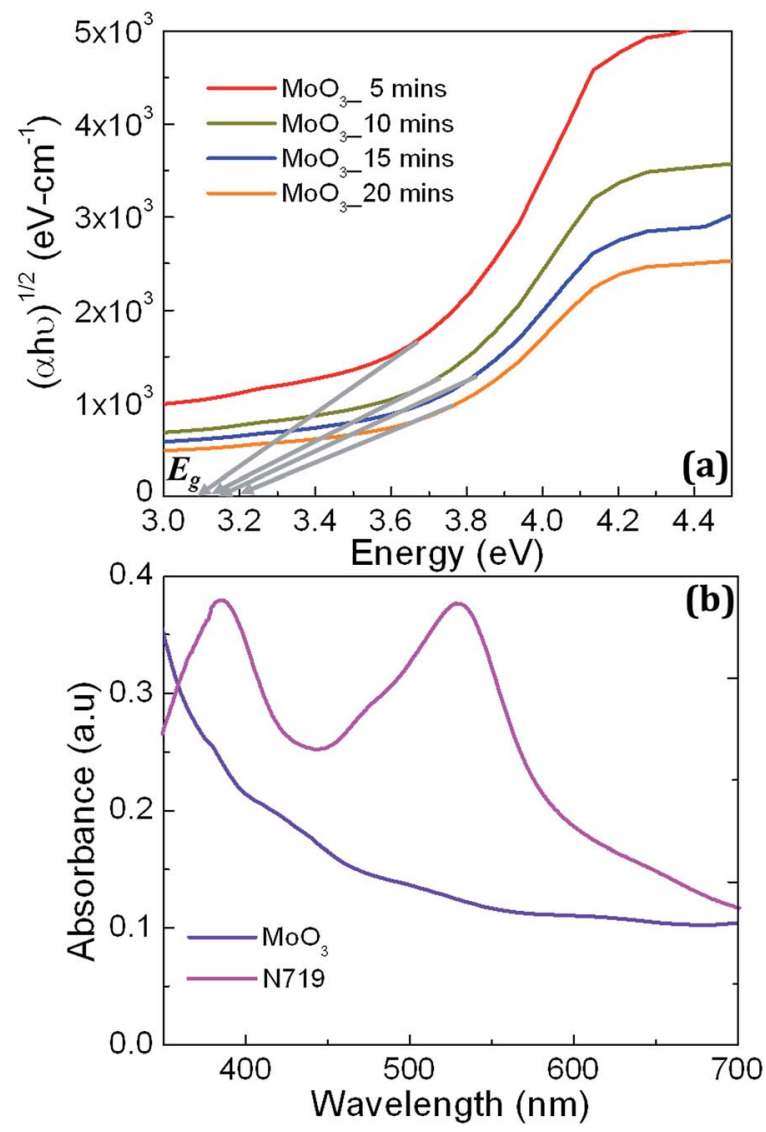

Fig. 4 (a) Optical bandgap values of all $\mathrm{MoO}_{3}$ samples from $(\alpha h \nu)^{1 / 2} v s$. energy of the incoming photons, (b) comparison of absorbance of $\mathrm{MoO}_{3}$ and $\mathrm{N719}$ dye to confirm the optical inactiveness of $\mathrm{MoO}_{3}$ in the spectral window of $350-700 \mathrm{~nm}$ in which N719 is optically active in terms of photo-absorption. 
a passive material component in DSSCs. It is expected to simply pass the visible spectral photons towards N719 at which major photonic absorption process occurs. Fig. 4(b) confirms $\mathrm{MoO}_{3}$ behaves optically inactive, in terms of absorption, in the spectral window of $350-800 \mathrm{~nm}$ where N719 is optically active to absorb the visible energy. The optical absorbance and transmittance characteristics with a bandgap value of $3.1 \mathrm{eV}$ support $\mathrm{MoO}_{3}$ as a potential RBL at $\mathrm{FTO} / \mathrm{TiO}_{2}$ interface to suppress the recombination loss in DSSCs. Further, the four samples considered in this work, deposited at 5, 10, 15 and 20 minutes, exhibit insignificant optical loss in the visible spectral range due to the wide bandgap which is an essential requirement for RBLs.

Fig. 5(a) shows $J-V$ characteristics of the DSSCs employed $\mathrm{MoO}_{3}$ RBLs deposited at different duration along with the reference DSSC which did not use any kind of RBLs and surface treatment. This work examines only the effect of $\mathrm{MoO}_{3}$ in the charge transport and recombination dynamics at the $\mathrm{FTO} / \mathrm{TiO}_{2}$ interface, so the reported DSSCs did not use any surface modifications on $\mathrm{TiO}_{2}$. All the reported photovoltaic parameters in this study were measured under standard AM1.5 illumination level. Table 1 lists the performance metrics of all DSSCs presented in Fig. 5(a). In general, $\mathrm{MoO}_{3}$ thin film RBLs at FTO/ $\mathrm{TiO}_{2}$ interface influences almost all photovoltaic parameters as can be observed in Fig. 5(a). It is very obvious that a dielectric layer across an electrical path of the photo-electrons will impede the transport characteristics. However, an optimum thickness of RBL is expected to facilitate the charge transport at $\mathrm{FTO} / \mathrm{TiO}_{2}$ interface. Fabrication steps, parameters such as $\mathrm{TiO}_{2}$ thickness
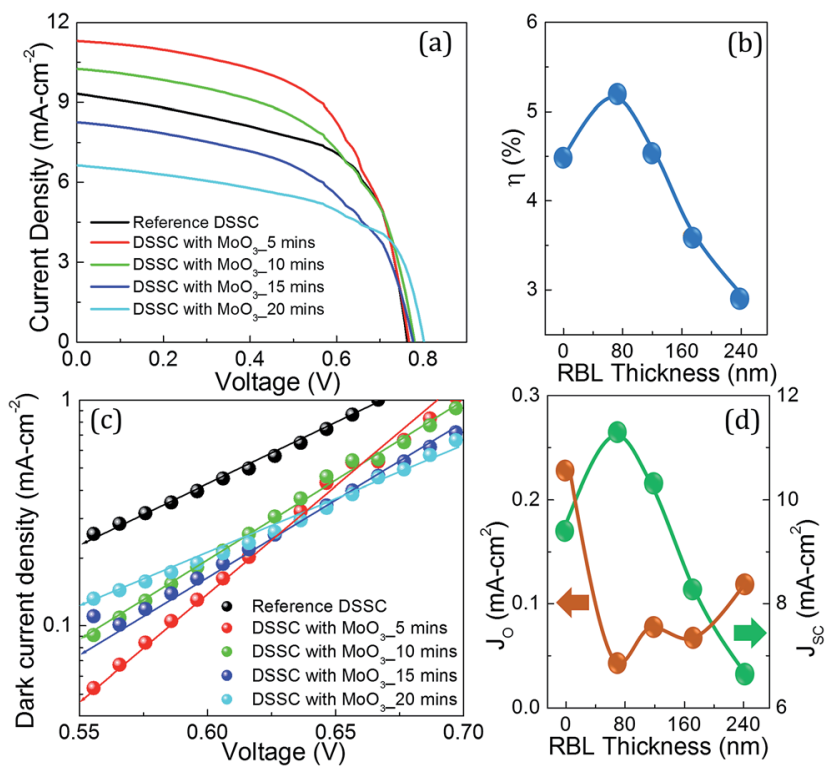

Fig. 5 (a) $J-V$ characteristics of the DSSCs employed $\mathrm{MoO}_{3}$ RBLs of different thickness showing a significant variation in $J_{\mathrm{SC}}$ values and (b) thickness dependent variation in efficiency of DSSCs showing the possibility of achieving better performance for an optimum thickness of $\mathrm{MoO}_{3}$. (c) Semi-log plot of dark J-V characteristics in the medium forward bias region used for fitting to get $J_{O}$ and (d) thickness dependency of $J_{O}$ and $J_{S C}$ values.
Table 1 Photovoltaic parameters measured under AM1.5 illumination condition

\begin{tabular}{lllll}
\hline DSSCs & $J_{\text {SC }}\left(\mathrm{mA} \mathrm{cm}^{2}\right)$ & $V_{\mathrm{OC}}(\mathrm{mV})$ & $\mathrm{FF}(\%)$ & $\eta(\%)$ \\
\hline Reference & 09.4 & 763 & 56.4 & 4.5 \\
5 min $\mathrm{MoO}_{3} \mathrm{RBL}$ & 11.3 & 766 & 59.4 & 5.2 \\
$10 \mathrm{~min} \mathrm{MoO}_{3} \mathrm{RBL}$ & 10.3 & 778 & 56.1 & 4.5 \\
$15 \mathrm{~min} \mathrm{MoO}_{3} \mathrm{RBL}$ & 08.3 & 776 & 56.7 & 3.6 \\
$20 \mathrm{~min} \mathrm{MoO}_{3} \mathrm{RBL}$ & 06.6 & 798 & 55.7 & 2.9 \\
\hline
\end{tabular}

and other conditions for all DSSCs reported in this study were exactly the same except the incorporation of $\mathrm{MoO}_{3}$ RBLs. We observed that a reference DSSC with no RBL exhibited photoconversion efficiency $(\eta)$ of $4.5 \%$. DSSC which used 5 minutes deposition of $\mathrm{MoO}_{3}$ resulted in $\eta$ of $5.2 \%$ with $J_{\mathrm{SC}}$ and $V_{\mathrm{OC}}$ of $11.3 \mathrm{~mA} \mathrm{~cm}^{-2}$ and $766 \mathrm{mV}$ respectively. As listed in Table 1, enhancement in the $J_{\mathrm{SC}}$ value suggests that 5 minutes deposition of $\mathrm{MoO}_{3}$ at $\mathrm{FTO} / \mathrm{TiO}_{2}$ interface favors the charge transport while other higher $\mathrm{MoO}_{3}$ thickness values suppressed the $J_{\mathrm{SC}}$ in DSSCs. DSSC with $\mathrm{MoO}_{3} \mathrm{RBL}$ deposited for 20 minutes resulted in $\eta$ of $2.9 \%$ which is $55 \%$ lower than that reference DSSC. These two $\mathrm{MoO}_{3}$ RBLs and their comparison with reference DSSC clearly confirms that photovoltaic performance can be increased by using an optimum thickness of $\mathrm{MoO}_{3}$ at $\mathrm{FTO} / \mathrm{TiO}_{2}$ interface. Fig. 5(b) shows thickness dependency of $\eta$ of the DSSCs with $\mathrm{MoO}_{3}$ RBL. The $\eta$ value corresponds zero thickness represents the reference DSSC. A deep drop in the $\eta$ value for increasing thickness was observed due to the reduction in the photo-electron transport through the RBL. While the change in $J_{\text {SC }}$ can directly be related to the photo-electron collection at the FTO electrode, $V_{\mathrm{OC}}$ represents the change in band alignment due to the incorporation of $\mathrm{MoO}_{3}$ at the $\mathrm{FTO} / \mathrm{TiO}_{2}$ interface. In general, $V_{\text {OC }}$ can be defined as the energy difference between redox potential of the electrolyte and the quasi-Fermi energy in the $\mathrm{TiO}_{2}$. In general, PVD processed thin films are considered to be denser materials than colloidal nanoparticles which results in efficient recombination blocking characteristic at the FTO/ $\mathrm{TiO}_{2}$ interface.

In general, dark saturation current density $\left(J_{\mathrm{O}}\right)$ in DSSCs represents electronic quality of interfaces where the electron transport process is impeded by various recombination dynamics. Single diode model equation is given by $J_{\mathrm{D}}=J_{\mathrm{O}^{-}}$ $\exp \left(q V / k T^{-1}\right)$, where $J_{\mathrm{D}}$ and $J_{\mathrm{O}}$ are current density and saturation current under dark condition. The parameters $q, V, k$ and $T$ represent electronic charge, applied bias, Boltzmann constant and operating temperature. Dark current of a solar cell must be suppressed as much as possible to achieve improved solar cell parameters under illumination. Thus, it is essential to obtain $J_{\mathrm{O}}$ as low as possible for DSSCs to get better $\eta$ values. Fig. 5(c) shows semi-log plot of dark current density vs. voltage in a medium forward bias region (0.55-0.7 V). The highly linear current density data set in semi-log plot confirm that they are exponential in linear scale. Single diode model was used to fit to extract the $J_{\mathrm{O}}$ and the values are shown in Fig. 5(d) with respect to RBL thickness values. We observed that 5 minutes deposited $\mathrm{MoO}_{3}$ suppressed the $J_{\mathrm{O}}$ very efficiently than other $\mathrm{MoO}_{3}$ RBLs 
and the reference DSSC. The $\mathrm{MoO}_{3}$ layer controlled the $J_{\mathrm{O}}$ value in the DSSC and resulted in lower $J_{\mathrm{D}}$ which led to improved performance under illumination, particularly $J_{\mathrm{SC}}$ values.

Nyquist and Bode phase plots of the reference and the DSSCs employing $\mathrm{MoO}_{3}$ RBLs are shown in Fig. 6(a and b) respectively. In general, the semicircles observed in $\mathrm{kHz}$ frequency range represent the charge transfer process at the $\mathrm{TiO}_{2} /$ electrolyte interfaces in DSSCs. The semicircles obtained from the Nyquist plots assure that the $\mathrm{MoO}_{3}$ RBLs introduced at $\mathrm{FTO} / \mathrm{TiO}_{2}$ interface significantly influenced the charge transfer process in the resulting DSSCs. The larger semicircles obtained for the DSSCs with 5 minutes coating of $\mathrm{MoO}_{3}$ RBL on FTO compared with reference DSSC confirming the recombination resistance between photo-generated electrons and holes at electrolyte is significantly improved due to the presence of $\mathrm{MoO}_{3}$. Also, the larger semicircle corresponding to the 5 minutes $\mathrm{MoO}_{3} \mathrm{RBL}$ at FTO/ $\mathrm{TiO}_{2}$ interface elucidates that charge transport process has been effectively facilitated by $\mathrm{MoO}_{3}$ compared to the reference DSSC. It is evident that electrolyte in DSSCs diffuse through the dye coated $\mathrm{TiO}_{2}$ nanoparticle layer to form the junction as a hole transport material candidate. However, this can lead to a direct electrical contact with FTO which is considered to be an electrical short. Nyquist plots obtained from the reference and DSSCs employing $\mathrm{MoO}_{3}$ RBLs show that such leaky charge transport pathways were effectively blocked by $\mathrm{MoO}_{3}$ RBLs. The Bode phase characteristics of the DSSCs shown in Fig. 6(b) depict that frequency values corresponding to the maximum phase angle shift towards lower values in case of DSSCs employing $\mathrm{MoO}_{3}$ RBLs compared to the reference DSSC. The low frequency shift observed in the DSSCs with $\mathrm{MoO}_{3}$ RBLs assures that charge transfer process occurs more efficiently compared with the reference DSSC. However, the optimum thickness value of $\mathrm{MoO}_{3}$ (5 minutes coating) resulting in higher $J_{\text {SC }}$ values compared to the reference DSSC while other DSSCs exhibit lower $J_{\mathrm{SC}}$ values due to the increased thickness of $\mathrm{MoO}_{3}$ which impede charge transport while they establish an energy barrier for recombination between photo-electrons and holes.

$\mathrm{TiCl}_{4}$ is commonly used in DSSCs to treat the surface of mesoporous $\mathrm{TiO}_{2}$ and FTO. The present work introduces physical vapor deposition based $\mathrm{MoO}_{3}$ as an efficient RBL in DSSCs and the results are further compared with $\mathrm{TiCl}_{4}$ treated FTO in
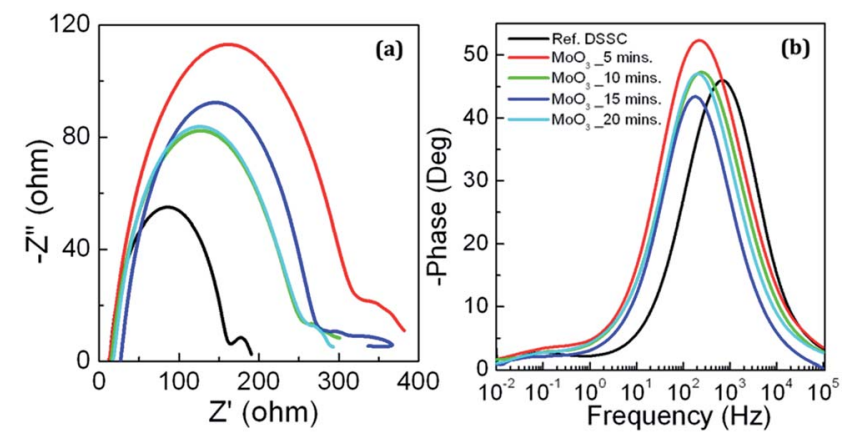

Fig. 6 (a) Nyquist and (b) Bode phase characteristics of the reference DSSC along with the DSSCs employing $\mathrm{MoO}_{3}$ RBLs. Legends are common for both (a) and (b).
DSSCs. Fig. 7(a) shows $J-V$ characteristics of two DSSCs with $\mathrm{TiCl}_{4}$ treated FTO electrodes showing very consistent photovoltaic characteristics under AM1.5 illumination. The $J-V$ characteristics of the two DSSCs are represented as cell 1 and 2 . This study utilized the same FTO, mesoporous $\mathrm{TiO}_{2}$, dye, electrolyte and Pt counter electrode used in the reference and $\mathrm{MoO}_{3}$ based DSSCs. The only difference is the $\mathrm{TiCl}_{4}$ treatment on FTO. The DSSC with FTO/ $/ \mathrm{TiCl}_{4}$ showed $J_{\mathrm{SC}}, V_{\mathrm{OC}}, \mathrm{FF}$ and $\eta$ of $5.5 \mathrm{~mA} \mathrm{~cm}{ }^{-2}, 808 \mathrm{mV}, 71.2 \%$ and $3.2 \%$ respectively. This study observed only the $V_{\mathrm{OC}}$ and FF were significantly improved by $\mathrm{TiCl}_{4}$ but overall performance remained lower than other DSSCs.

Further, EIS studies were performed on the DSSCs with $\mathrm{TiCl}_{4}$ treated FTO to examine the Nyquist and Bode phase plots shown in Fig. 7(b and c) respectively. The semi-circles obtained from the DSSC with $\mathrm{TiCl}_{4}$ treated DSSCs shown in Fig. 7(b) are smaller than the one obtained from $\mathrm{MoO}_{3} \mathrm{RBL}(5$ minutes coating) assures the recombination resistance is smaller and this can be correlated to the difference in the blocking capability between $\mathrm{TiCl}_{4}$ and $\mathrm{MoO}_{3}$. The present study did not use any light scattering layers or surface treatment on mesoporous $\mathrm{TiO}_{2}$ to examine specifically the $\mathrm{FTO} / \mathrm{TiO}_{2}$ interface. Thus, photovoltaic performance of the DSSCs reported in the present study is relatively lower compared to previous report. ${ }^{41}$

In general, incorporating $\mathrm{MoO}_{3}$ at $\mathrm{FTO} / \mathrm{TiO}_{2}$ interface influences the charge transport at the $\mathrm{FTO} / \mathrm{TiO}_{2}$ interface while acts as a barrier for recombination between photo-generated charge carriers and hole present in the electrolyte that diffuses through the mesoporous $\mathrm{TiO}_{2}$ nanoparticle layer. The charge transport characteristics can further be explained through the energy level alignment in the DSSCs with and without $\mathrm{MoO}_{3}$ RBLs. Fig. 8(a)

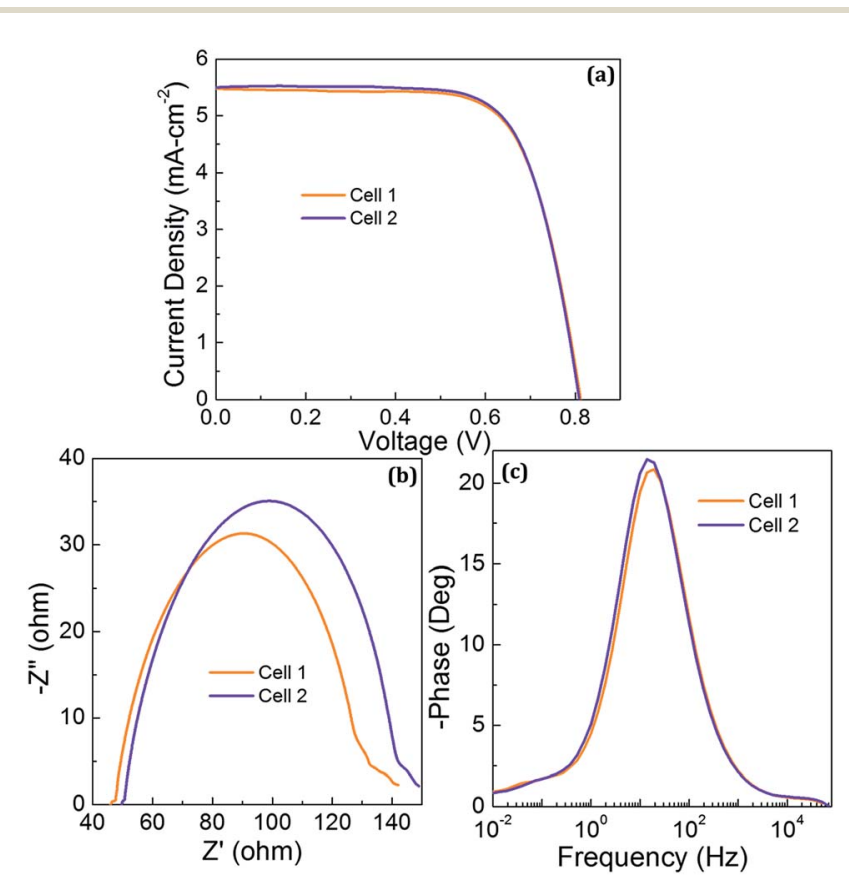

Fig. 7 (a) $J-V$ characteristics of the two DSSCs employed $\mathrm{TiCl}_{4}$ treatment on FTO (b) Nyquist and (c) Bode phase characteristics of the two DSSCs. 


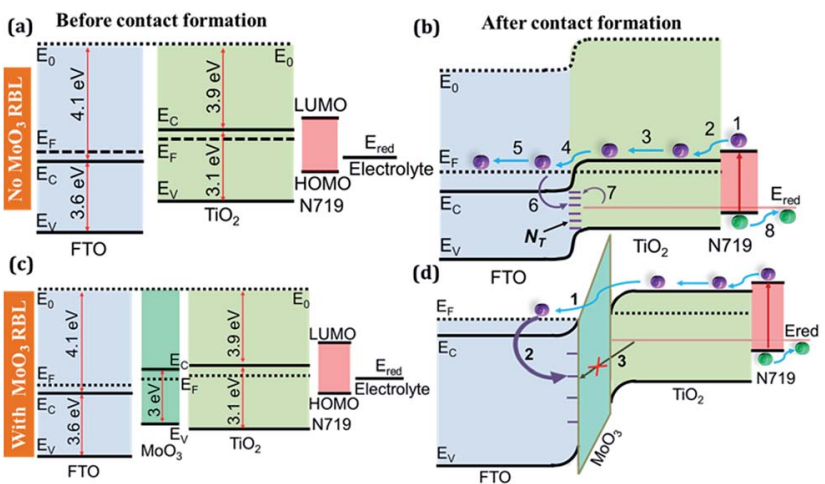

Fig. 8 (a) Energy band diagrams of the $\mathrm{FTO}, \mathrm{TiO}_{2}, \mathrm{~N} 719$ and electrolyte before contact formation and (b) energy level alignment at FTO/ $\mathrm{TiO}_{2} / \mathrm{N} 719 /$ electrolyte interfaces after contact formation. (c) Energy levels representing $\mathrm{MoO}_{3} \mathrm{RBL}$ at $\mathrm{FTO} / \mathrm{TiO}_{2}$ interface and (d) the alignment facilitating the charge transport through $\mathrm{MoO}_{3}$.

shows the energy band diagrams of the FTO, $\mathrm{TiO}_{2}, \mathrm{~N} 719$ and electrolyte before making the contact under thermal equilibrium (no exciton generation). Fig. 8(b) shows the alignment of energy levels of the $\mathrm{FTO} / \mathrm{TiO}_{2} / \mathrm{N} 719 /$ electrolyte interfaces and major physical processes occur under illumination. Process 1 , 2, 3, 4 and 5 represent exciton generation in N719, electron injection by $\mathrm{N} 719$ to $E_{\mathrm{C}}$ of the $\mathrm{TiO}_{2}$, diffusion, collection at FTO and transport in the FTO respectively. The incoming solar photons enter into the DSSCs through FTO and $\mathrm{TiO}_{2}$. Optical bandgap values of FTO and $\mathrm{TiO}_{2}$ are $3.6 \mathrm{eV}$ and $3.1 \mathrm{eV}$ respectively which simply allow the visible energy spectral photons without any significant loss. Photonic interaction with N719 results in optical excitation and generates excitons as illustrated in process 1 . The photo-generated electrons are injected into the $E_{\mathrm{C}}$ of the $\mathrm{TiO}_{2}$ nanoparticles from the lowest unoccupied molecular orbital (LUMO) of N719. Diffusion of photo-electrons in the bulk of mesoporous $\mathrm{TiO}_{2}$ leads charge transport to reach the Fermi level $\left(E_{\mathrm{F}}\right)$ of FTO electrode.

Mesoporous $\mathrm{TiO}_{2}$ is preferred to adsorb more dye molecules; however, the porosity allows the liquid electrolyte to diffuse into the bulk to make $\mathrm{TiO}_{2} / \mathrm{N} 719 /$ electrolyte junction at which the electron-hole transport process starts after exciton dissociation. While the electrolyte diffuses into the bulk $\mathrm{TiO}_{2}$, the porosity of $\mathrm{TiO}_{2}$ offers a chance to make a direct electrical contact with the FTO which is considered to be an electrical short in DSSCs. Further, the defect states that are associated with $\mathrm{FTO} / \mathrm{TiO}_{2}$ interface also actively participate in the electron capture process which can effectively trap photo-generated electrons at the FTO/ $/ \mathrm{TiO}_{2}$ interface as represented by process 6 in Fig. 8(b). The electron capture process is expected to be followed by a hole capture process 7 as the electrolyte is in the vicinity of those defect states which capture photo-electrons. The two processes, electron-hole capture, lead to interfacial recombination mediated by the interfacial defects at $\mathrm{FTO} / \mathrm{TiO}_{2}$ interface. Fig. 8(c) shows band diagrams of FTO, $\mathrm{TiO}_{2}$, N719 and electrolyte along with $\mathrm{MoO}_{3} \mathrm{RBL}$ at $\mathrm{FTO} / \mathrm{TiO}_{2}$ interface. Fig. 8(d) shows the energy level alignment at $\mathrm{FTO} / \mathrm{MoO}_{3} / \mathrm{TiO}_{2} / \mathrm{N} 719 /$ electrolyte interfaces. From the illuminated $J-V$ characteristics of the
DSSCs, it is confirmed that $\mathrm{MoO}_{3}$ RBLs changed in $\mathrm{FTO} / \mathrm{TiO}_{2}$ interface and thus the density and activity of the interfacial defects. The $\mathrm{MoO}_{3}$ layers acts as an energy barrier between photo-electrons and the hole present at the redox potential of the hole transport electrolyte. Thus, electron-hole recombination is significantly prohibited by incorporating $\mathrm{MoO}_{3}$ at FTO/ $\mathrm{TiO}_{2}$ interface. It is expected that if $\mathrm{MoO}_{3} \mathrm{RBL}$ establishes an energy barrier between the photo-electrons and the holes at the redox potential of the hole transport layer, then the $J_{\mathrm{SC}}$ under illumination can be increased significantly by suppressing the interfacial recombination mediated by the defect states at FTO/ $\mathrm{TiO}_{2}$ interface as shown in Fig. 8(d).

In general, process 1 is sensitive to the thickness of such interfacial layers and thus obtained results confirm the $J_{\mathrm{SC}}$ decreased for further increase in the $\mathrm{MoO}_{3}$. Process 2 and 3 represent the electron and hole capture processes respectively. These two processes can happen effectively when there is no RBL and these two are suppressed by the RBL with an optimum thickness to facilitate the process 1 while 2 and 3 are prohibited.

\section{Conclusions}

Performance limiting interfacial recombination at $\mathrm{FTO} / \mathrm{TiO}_{2}$ interface can be suppressed by coating $\mathrm{MoO}_{3}$ layers on FTO by reactive DC sputtering. There is no significant optical loss in $\mathrm{MoO}_{3}$ films, even for 20 minutes deposition. The $15 \%$ enhancement in the performance of DSSC is attributed to the effect of $\mathrm{MoO}_{3}$ on electron-hole capture processing which were significantly controlled for an optimum growth of 5 minutes. Further increase in the thickness of $\mathrm{MoO}_{3}$ impedes the charge transport process at $\mathrm{FTO} / \mathrm{TiO}_{2}$ interface and affects the DSSC performance. The presented work leads to the establishment of defect engineered material interfaces to progress the research and developmental works in DSSCs.

\section{Conflicts of interest}

The authors declare no conflict of interest.

\section{Acknowledgements}

We thank Department of Science and Technology, Government of India for financial support through Solar Energy Research Initiative. The authors would like to thank Dr Dhamodaran Santhanagopalan for providing Mo sputter target through his Ramanujan Fellowship and AEPL for PVD cluster facility.

\section{References}

1 M. Wright and A. Uddin, Sol. Energy Mater. Sol. Cells, 2012, 107, 87-111.

2 S. Günes and N. S. Sariciftci, Inorg. Chim. Acta, 2008, 361, 581-588.

3 P. Poudel and Q. Qiao, Nanoscale, 2012, 4, 2826-2838.

4 B. O'regan and M. Grfitzel, Nature, 1991, 353, 737-740. 
5 J. M. Kroon, N. J. Bakker, H. J. P. Smit, P. Liska, K. R. Thampi, P. Wang, S. M. Zakeeruddin, et al., Prog. Photovoltaics, 2007, 15, 1-18.

6 T. G. Deepak, G. S. Anjusree, S. Thomas, T. A. Arun, S. V. Nair and A. S. Nair, RSC Adv., 2014, 4, 17615-17638.

7 R. Harikisun and H. Desilvestro, Sol. Energy, 2011, 85, 11791188.

8 N. Kato, K. Higuchi, H. Tanaka, J. Nakajima, T. Sano and T. Toyoda, Sol. Energy Mater. Sol. Cells, 2011, 95, 301-305.

9 A. Hinsch, J. M. Kroon, R. Kern, I. Uhlendorf, J. Holzbock, A. Meyer and J. Ferber, Prog. Photovoltaics, 2001, 9, 425-438.

10 M. I. Asghar, K. Miettunen, J. Halme, P. Vahermaa, M. Toivola, K. Aitola and P. Lund, Energy Environ. Sci., 2010, 3, 418-426.

11 D. Kuang, C. Klein, S. Ito, J.-E. Moser, R. Humphry-Baker, N. Evans, F. Duriaux, C. Graetzel, S. M. Zakeeruddin and M. Grätzel, Adv. Mater., 2007, 19, 1133-1137.

12 P. Wang, S. M. Zakeeruddin, R. Humphry-Baker, J. E. Moser and M. Grätzel, Adv. Mater., 2003, 15, 2101-2104.

13 M. Grätzel, Prog. Photovoltaics, 2000, 8, 171-185.

14 K. Shankar, G. K. Mor, H. E. Prakasam, S. Yoriya, M. Paulose, O. K. Varghese and C. A. Grimes, Nanotechnology, 2007, 18, 065707.

15 S. V. Nair, A. Balakrishnan, K. R. V. Subramanian, A. M. Anu, A. M. Asha and B. Deepika, Bull. Mater. Sci., 2012, 35, 489493.

16 B. Tan and Y. Wu, J. Phys. Chem. B, 2006, 110, 15932-15938.

17 E. Ghadiri, N. Taghavinia, S. M. Zakeeruddin, M. Grätzel and J.-E. Moser, Nano Lett., 2010, 10, 1632-1638.

18 D. Chen, F. Huang, Y.-B. Cheng and R. A. Caruso, Adv. Mater., 2009, 21, 2206-2210.

19 S. Agarwal, M. Kevin, A. S. W. Wong, C. K. N. Peh, V. Thavasi and G. W. Ho, ACS Appl. Mater. Interfaces, 2010, 2, 18441850.

20 Z.-Q. Li, Y. Ding, L.-E. Mo, L.-H. Hu, J.-H. Wu and S.-Y. Dai, ACS Appl. Mater. Interfaces, 2015, 7, 22277-22283.

21 M. Safdari, P. W. Lohse, L. Häggman, S. Frykstrand, D. Högberg, M. Rutland, R. A. Asencio, et al., RSC Adv., 2016, 6, 56580-56588.

22 H. Cheema and J. H. Delcamp, ACS Appl. Mater. Interfaces, 2017, 9, 3050-3059.
23 A. Yella, S. Mathew, S. Aghazada, P. Comte, M. Grätzel and M. K. Nazeeruddin, J. Mater. Chem. C, 2017, 5, 2833-2843.

24 J. Van de Lagemaat and A. J. Frank, J. Phys. Chem. B, 2000, 104, 4292-4294.

25 Q. Yu, Y. Wang, Z. Yi, N. Zu, J. Zhang, M. Zhang and P. Wang, ACS Nano, 2010, 4, 6032-6038.

26 A. Burke, S. Ito, H. Snaith, U. Bach, J. Kwiatkowski and M. Grätzel, Nano Lett., 2008, 8, 977-981.

27 N. Huang, Y. Liu, T. Peng, X. Sun, B. Sebo, Q. Tai, H. Hu, B. Chen, S.-S. Guo and X. Zhao, J. Power Sources, 2012, 204, 257-264.

28 B. Bills, M. Shanmugam and M. Farrokh Baroughi, Thin Solid Films, 2011, 519, 7803-7808.

29 S. Lee, J. H. Noh, H. S. Han, D. K. Yim, D. H. Kim, J.-K. Lee, J. Y. Kim, H. S. Jung and K. S. Hong, J. Phys. Chem. C, 2009, 113, 6878-6882.

30 W.-Q. Zhou, Y.-M. Lu, C.-Z. Chen, Z.-Y. Liu and C.-B. Cai, J. Inorg. Mater., 2011, 26, 819-822.

31 H. Choi, C. Nahm, J. Kim, J. Moon, S. Nam, D.-R. Jung and B. Park, Curr. Appl. Phys., 2012, 12, 737-741.

32 T. Matsushima, Y. Kinoshita and H. Murata, Appl. Phys. Lett., 2007, 91.25, 253504.

33 H. Lee, S. W. Cho, K. Han, P. E. Jeon, C.-N. Whang, K. Jeong, K. Cho and Y. Yi, Appl. Phys. Lett., 2008, 93, 279.

34 J. Meyer and A. Kahn, J. Photonics Energy, 2011, 1, 011109.

35 D. Mutschall, K. Holzner and E. Obermeier, Sens. Actuators, $B$, 1996, 36, 320-324.

36 K. Gesheva, A. Kostadinka and T. Ivanova, Chem. Vap. Deposition, 2006, 12, 231-238.

37 F. Cheng, Y. Wu, Y. Shen, X. Cai and L. Li, $R S C A d v .$, 2017, 7, 37952-37958.

38 C. Julien, A. Khelfa, O. M. Hussain and G. A. Nazri, J. Cryst. Growth, 1995, 156, 235-244.

39 O. M. Hussain, K. S. Rao, K. V. Madhuri, C. V. Ramana, B. S. Naidu, S. Pai, J. John and R. Pinto, Appl. Phys. A: Mater. Sci. Process., 2002, 75, 417-422.

40 A. K. Prasad, D. J. Kubinski and P. I. Gouma, Sens. Actuators, B, 2003, 93, 25-30.

41 S. Ito, T. N. Murakami, P. Comte, P. Liska, C. Grätzel, M. K. Nazeeruddin and M. Grätzel, Thin Solid Films, 2008, 516, 4613-4619. 